\title{
Studying chaos in carbon-dioxide lasers in a graduate level optical laboratory
}

Emmanuil Rabinovich, Jacek Kowalski, Chris Littler

Emmanuil M. Rabinovich, Jacek M. Kowalski, Chris L. Littler, "Studying chaos in carbon-dioxide lasers in a graduate level optical laboratory," Proc. SPIE 2525, 1995 International Conference on Education in Optics, (13 October 1995); doi: 10.1117/12.224060

SPIE Event: SPIE's 1995 International Symposium on Optical Science, Engineering, and Instrumentation, 1995, San Diego, CA, United States 


\title{
Studying chaos in carbon-dioxide lasers in a graduate level optical laboratory
}

\author{
E. M. Rabinovich ${ }^{1}$, J. M. Kowalski², C. L. Littler ${ }^{2}$
}

1) Advanced Isotope Technologies, Incorporated, P.O. Box 5073, Denton, Texas 76203-5073, e-mail : emrabin@aol.com

2) Department of Physics, University of North Texas, Denton, Texas 76203, e-mail : jmk0002@jove.acs.unt.edu

\begin{abstract}
Carbon-dioxide lasers are well-known example of optical systems, which can exhibit complex transitions from the $\mathrm{CW}$ to the chaotic regime in the intensity caused by the modulation of various system parameters. They can demonstrate classical bifurcation scenarios with period doubling, bistability, and chaotic attractors .

We report on a new method of observing chaos in carbon-dioxide lasers where the simple technique of modulation of the optical feedback parameters is applied. In this approach the parameters of the carbon-dioxide laser itself remain unchanged and modulation is carried out externally. This leads to a system which can be easily assembled and studied in a graduate laboratory. Experimental results show the presence of stable periodic orbits of different periods, bistability of periodic orbits and chaotic attractors with complex transition scenarios, providing a wealth of examples for the student to investigate.
\end{abstract}

Key words : carbon-dioxide laser, chaos, period doubling, bifurcations, optical feedback, $\mathrm{CO}_{2}$ laser parameter modulation, periodic orbit, attractor, bistability

\section{INTRODUCTION AND BACKGROUND}

The transition from regular to irregular behavior in nonlinear dynamical optical systems has been a topic of both experimental and theoretical interest for over two decades. The observation of nonlinear dynamical phenomena and chaos has become a very important issue during last years from both a scientific and practical point of view and thus must be a subject of education in optics. In the context of laser systems one is interested, for example, in the practical problem of the control of the onset of chaos and in the decrease of dynamical noise in the laser output intensity.

It is well known that $\mathrm{CO}_{2}$ lasers can demonstrate classical transition scenarios to chaos (such as period doubling cascade, for instance) and therefore are a very good system for studying chaos. To investigate the chaos onset mechanisms a number 
of laser configurations in which chaos is induced in the output have been studied, e.g., a $\mathrm{CO}_{2}$ laser with internal harmonic modulation of the loss parameter ${ }^{1-4}, \mathrm{CO}_{2}$ laser with harmonic modulation of the pumping current ${ }^{5,6}$, a single $\mathrm{CO}_{2}$ lasers with delayed opto-electronic feedback ${ }^{7-9}$, and two or more optically coupled lasers $6,10,11$.

It is also known that $\mathrm{CO}_{2}$ lasers are very sensitive to radiation feedback. In this work the extreme sensitivity of $\mathrm{CO}_{2}$ lasers to optical feedback is employed to induce dynamical instabilities. We report here on the experimental and theoretical results concerning instabilities in $\mathrm{CO}_{2}$ lasers induced by an optical phase modulation of the back reflected signal. The above mentioned phase modulation was implemented by periodically varying either geometrical feedback length or the optical feedback length by means of a refractive index modulation using an electro-optical modulator (EOM). This new scheme of observing chaos in carbondioxide lasers doesn't require EOM accommodation inside of laser resonator and thus is significantly simpler than traditional schemes ${ }^{1-4}$. Such scheme can thus be easily implemented in a graduate level optical laboratory.

\section{EXPERIMENTAL PROCEDURE}

A schematic of the experimental setup is shown in Fig.1. In this scheme an axial

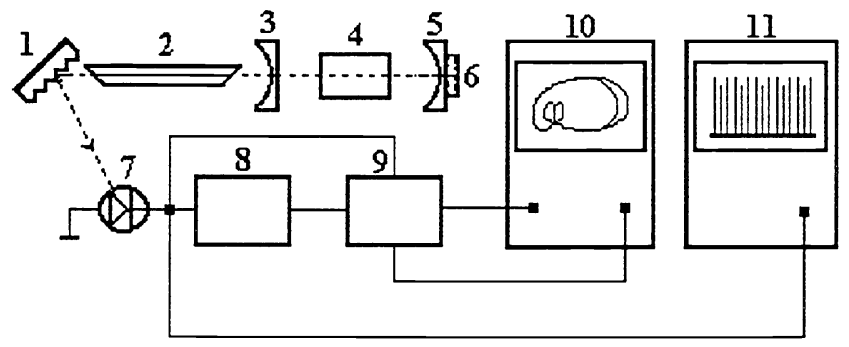

Figure 1

Experimental setup for obtaining chaos in a $\mathrm{CO}_{2}$ laser: 1. diffraction grating; 2 . $\mathrm{CO}_{2}$ laser tube; 3. output mirror; 4. electro-optical modulator (EOM); 5. additional mirror; 6. piezo-ceramic drive; 7. liquid nitrogen cooled $\mathrm{HgCdTe}$ detector; 8. delay line; 9. digitizer; 10. oscilloscope; 11. spectrum analyzer. 
flowing gas $\mathrm{CO}_{2}$ laser operating at a wave length of $\lambda=10.6 \mu \mathrm{m}$ was used. This laser has a resonant cavity $2.5 \mathrm{~m}$ long formed by a diffraction grating (1) and mirror (3), the diameter of the discharge tube (2) is $6 \mathrm{~mm}$, and the pressure of the conventional gas mixture varies from 15 to $20 \mathrm{mmHg}$. An additional mirror (5) provides the radiation feedback into the active region. Mirror (5) is mounted on a piezoelectric ceramic drive (6) allowing static tuning and/or periodic modulation of the external resonator length. The distance between mirrors (3) and (5) does not exceed $30 \mathrm{~cm}$. The laser intensity is measured by a $\mathrm{HgCdTe}$ detector (7) which monitor part of the radiation reflected from the diffraction grating (1). The intensity signal from the detector and its time-delayed copy are fed into an oscilloscope (10) operating in the $x-y$ mode. The delay line (8) has a constant delay of $2.5 \mu \mathrm{s}$. This simple scheme allows real time observations of the twodimensional projections of the system trajectories in the space of delayed signals.

We propose to organize the experiments into two major series following the approach we used when working with the system.

In the first series of experiments, (Section 3.1), the optical phase of the backreflected emission was modulated by using an electro-optical modulator (EOM) with a quarter wave voltage of $2.2 \mathrm{KV}$. The EOM was a Cd-Te crystal having an active length of $48 \mathrm{~mm}$, and an aperture diameter of $3 \mathrm{~mm}$. The voltage applied to the EOM had a DC component of $1.5 \mathrm{KV}$ and the amplitude and frequency of the modulated signal varied within $100-900 \mathrm{~V}$ and $50-85 \mathrm{KHz}$, respectively.

In the second series of experiments, discussed in Section 3.2, the phase of the feedback radiation was modulated by the induced oscillations of the feedback providing mirror (5) mounted on a piezoelectric drive. The driving frequencies varied from $30 \mathrm{KHz}$ to $60 \mathrm{KHz}$. The frequency range of these drives is centered on the relaxational frequencies of the laser system used. The largest amplitude of the external mirror oscillations was of the order of $1 \mu \mathrm{m}$ for the lowest driving frequencies and estimated as not exceeding $0.5 \mu \mathrm{m}$ for the highest driving frequencies used. In both series of experiments the amount of optical feedback radiation was measured to be less than $10 \%$.

\section{EXPERIMENTAL RESULTS}

\subsection{An optical phase modulation by means of EOM}

The results presented in Fig. 2 were obtained by varying the static part of the loss coefficient while all other laser parameters remained unchanged. The static part of the loss coefficient was varied by changing the angle of the output mirror of the laser. In the right column of Fig. 2 the frequency spectra are displayed, the middle column shows the respective phase portraits (Intensity $(t)$ vs Intensity $(t+\tau)$ ) and finally the left column contains the corresponding time series. These spectra were recorded at a constant frequency of $83 \mathrm{KHz}$ and a modulation amplitude of $150 \mathrm{~V}$. 
For relatively low levels of losses the laser intensity follows the driving signal with

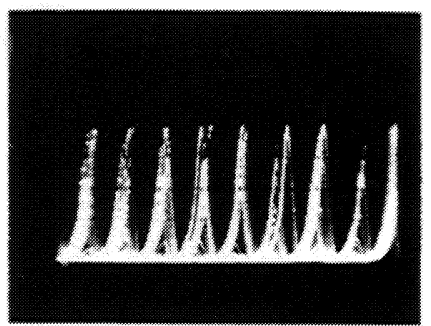

m
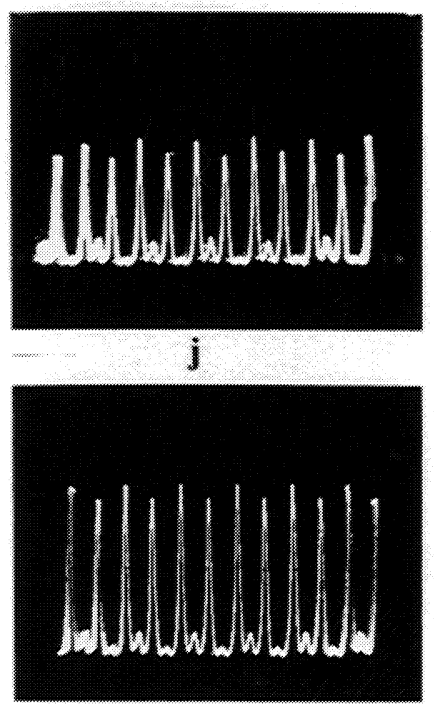

$\mathbf{g}$

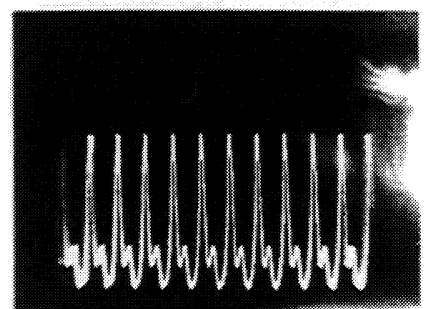

d

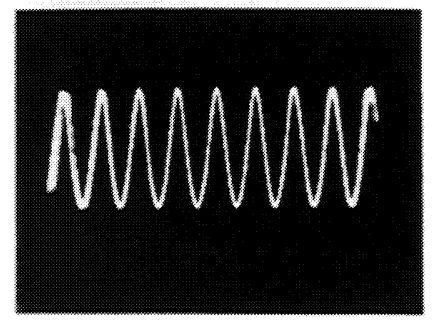

a

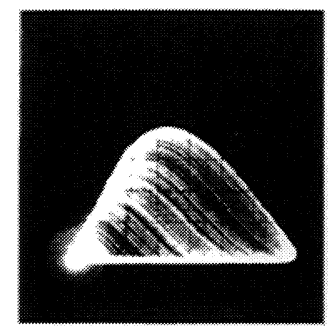

$\mathbf{n}$

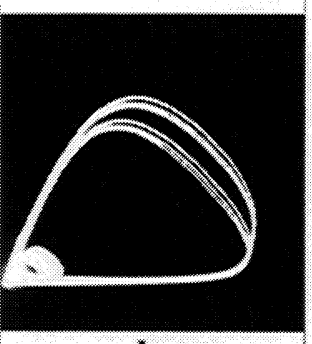

k

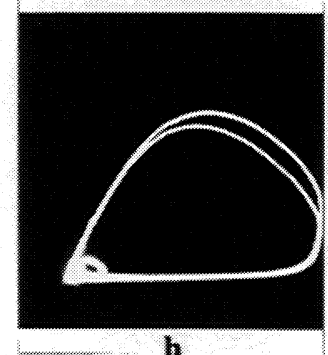

h

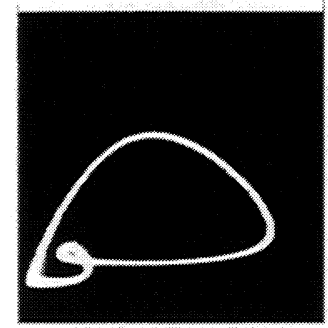

e

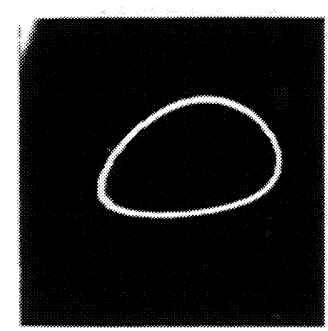

b

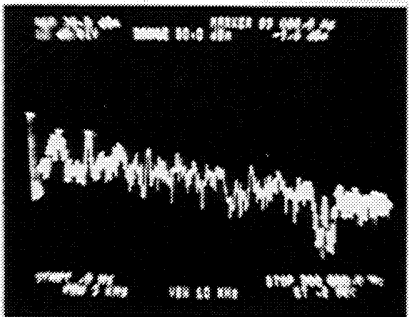

0

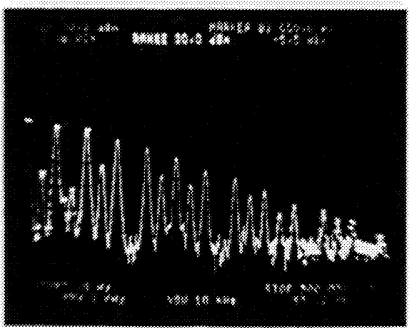

1

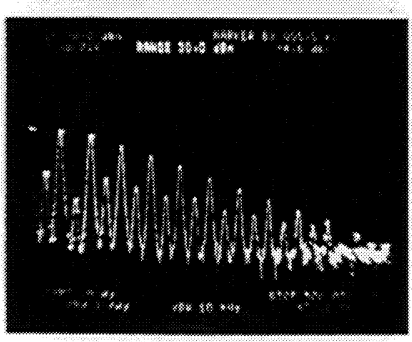

i

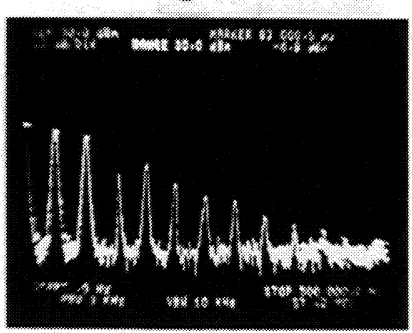

f

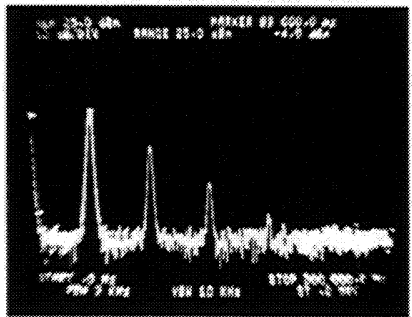

c

Figure 2

A representative sequence of laser states for a $\mathrm{CO}_{2}$ laser obtained by varying the static part of the loss coefficient while all other laser parameters were unchanged. 
a pronounced second and third harmonic present (Fig. 2c). As the losses are increased, the laser output becomes more and more structured and period 2 oscillations are observed as shown in Figs. 2d,e,f. Subsequently, period 4 is obtained for increasing losses as shown in Figs. 2g,h,i. Similar results were obtained for period 8 Figs. $2 \mathrm{j}, \mathrm{k}, 1$. Finally the frequency spectrum of the laser output changes into a broad spectrum with a rise in the floor level. This is a definitive indication that the system is driven into chaos, as shown in Figs. $2 \mathrm{~m}, \mathrm{n}, \mathrm{o}$. The chaotic attractor (Fig. 2n) has a characteristic shape typical for chaotic states obtained at other driving frequencies.

The bifurcation diagram presented in Fig. 3 was obtained by varying the amplitude

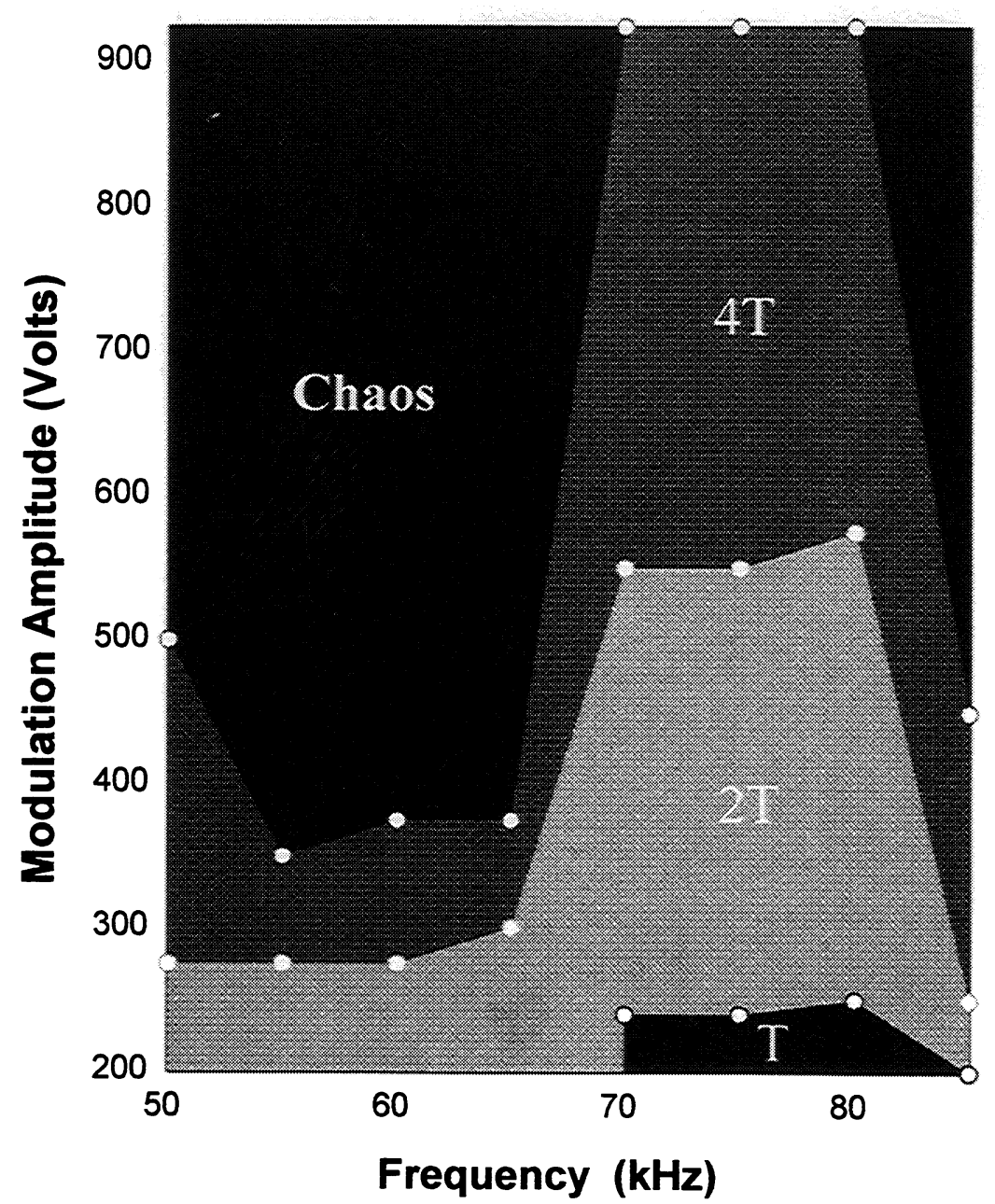

Figure 3

A bifurcation diagram in the (driving frequency, modulation amplitude) parameter space for a $\mathrm{CO}_{2}$ laser with a phase driven by an electro-optical modulator and with a fixed static part of the intensity loss coefficient . 
of ac voltage driving the EOM at a given frequency. The sequence of period doubling bifurcation obtained changing the amplitude of ac voltage, mentioned above, was identical to that obtained by varying the static part of the loss coefficient as described in the previous paragraph. In this case the modulation amplitude was varied while keeping all other parameters of the laser constant. The results obtained by this method are presented in Fig. 3 in the form of a complete bifurcation diagram. The bifurcation diagram clearly indicates that there is a broad range of frequencies in which the laser system could be driven into instability and chaos.

The coexistence of two stable periodic orbits is shown in Fig. 4. Transitions between two orbits of different periods are shown in Figs. 4 a,b. The corresponding frequency spectrum (Fig. $4 \mathrm{~b}$ ) contains the fundamental frequencies of both orbits. Similar bistabilities were reported previously ${ }^{1,3}$ with transitions between orbits of period 's 3 and 4.
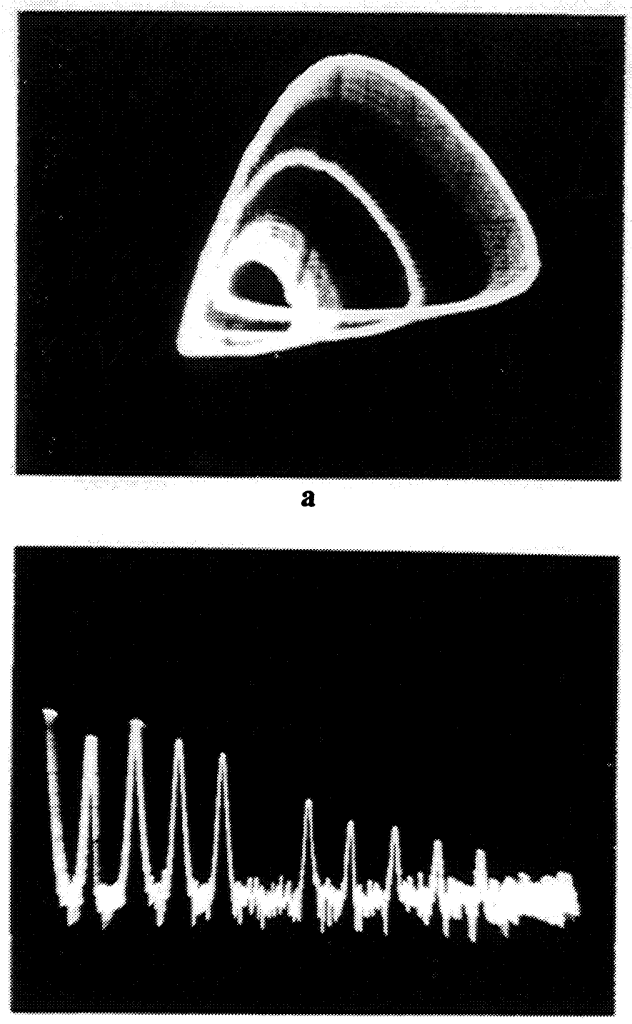

b

Figure 4

Bistability in $\mathrm{CO}_{2}$ laser with externally modulated optical feedback. EOM is used for optical phase modulation : a- phase portrait, b-spectrum ; driving frequency $f_{\mathrm{d}}=84 \mathrm{KHz}$. 


\subsection{An optical feedback length modulation by means of vibrating minor}

Instabilities and chaos induced by a vibrating mirror are presented in Fig. 5. The period doubling sequence, bifurcations, and attractor shapes obtained in this series of tests are very similar to those presented in section 3.1 for the optical phase modulation by EOM.

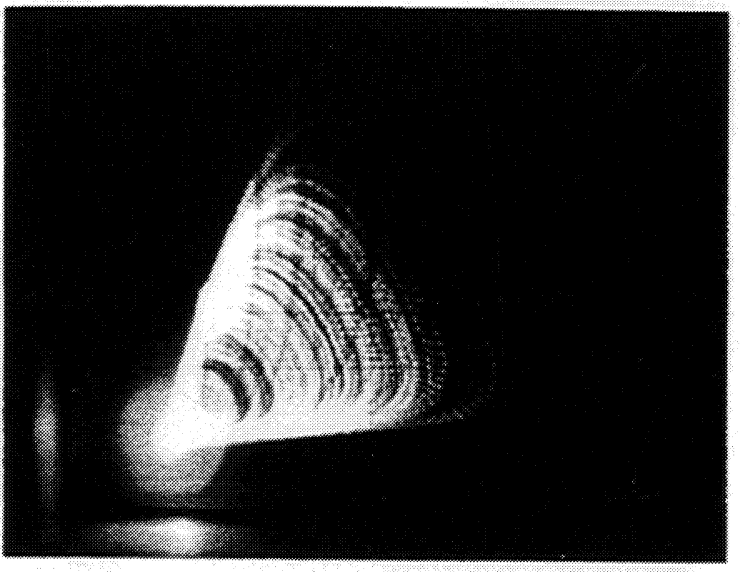

a

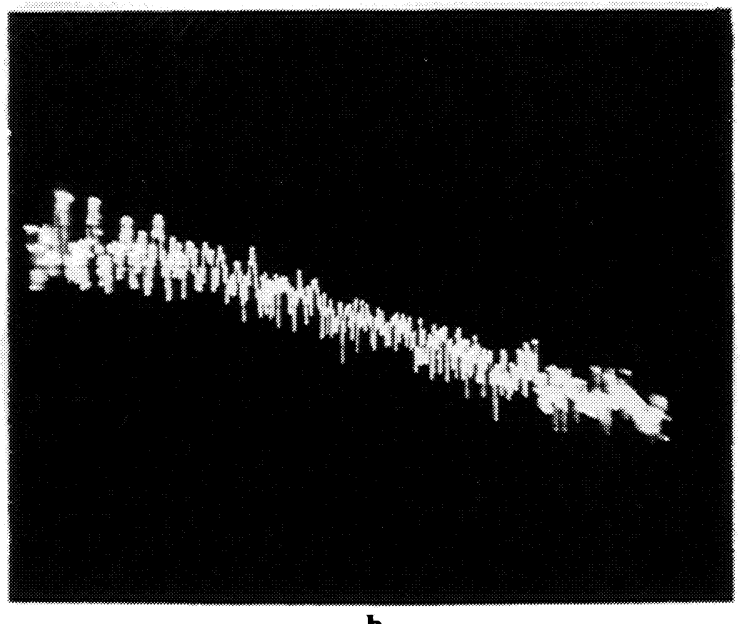

b

Figure 5

Chaos induced by a vibrating mirror, a- phase portrait; b- spectrum, $f_{\mathrm{d}}=30 \mathrm{KHz}$. 
Fig. 6 shows the coexistence of two period 1 orbits of different amplitudes and the same frequency. This is evidenced by the frequency spectrum shown in Fig. $6 \mathrm{~b}$ with fundamental frequency and higher harmonics.
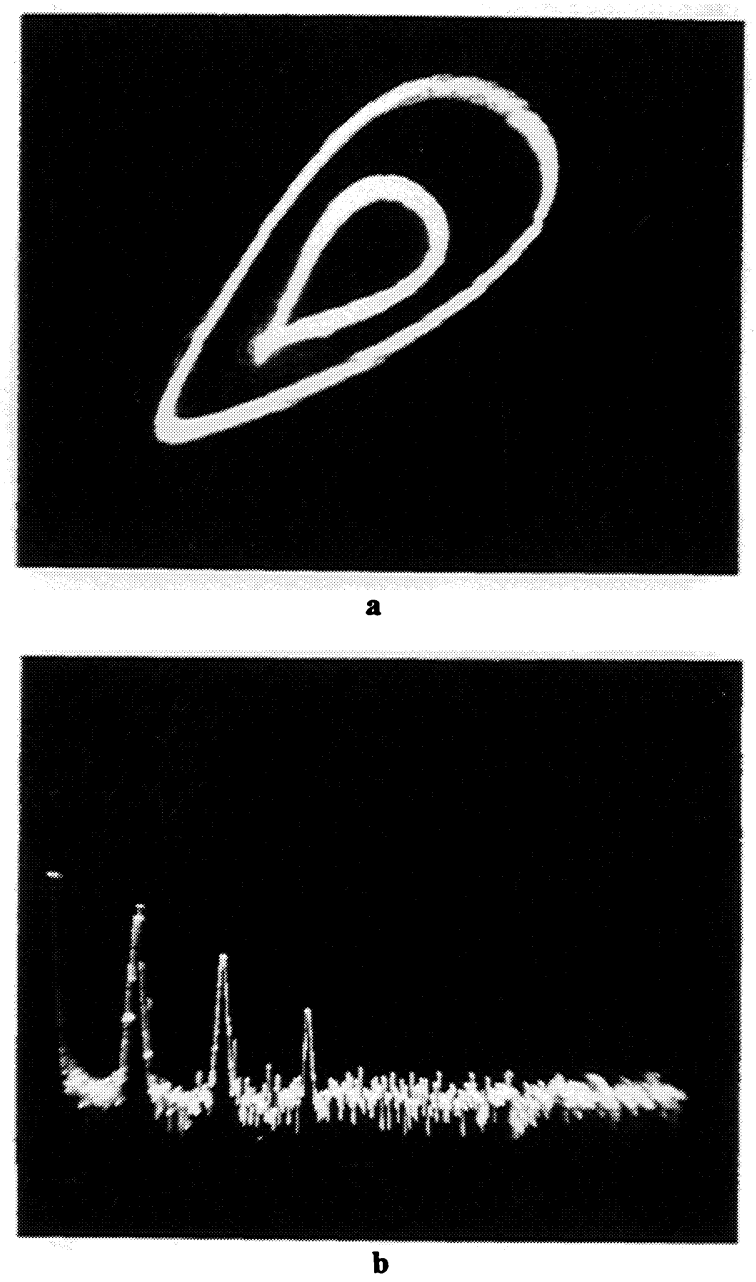

Figure 6

Bistability in $\mathrm{CO}_{2}$ laser with modulating length of feedback by vibrating mirror a- phase portrait, b-spectrum, $f_{\mathrm{d}}=60 \mathrm{KHz}$. 


\section{SUMMARY AND CONCLUSIONS}

We have shown that a $\mathrm{CO}_{2}$ laser can be driven into chaotic state by using a very simple method where one modulates externally the feedback of laser radiation.

Two approaches were used to drive the laser into chaotic state. In the first setup an EOM is used to modulate optical phase of re-injected signal, and the second one modulating the length ( and, of course, the phase) of the optical feedback. Both of these methods produced instabilities and chaos through period doubling cascades for a broad range of modulation frequencies. Uirder certain conditions the system also exhibits bistability. Both methods of chaos observation in carbondioxide laser can be successfully used for many types of low power $\mathrm{CO}_{2}$ lasers to study of nonlinear dynamical phenomenon in a graduate student laboratory.

The results of this work are very similar to those obtained for a $\mathrm{CO}_{2}$ laser with modulated intercavity losses ${ }^{1-4}$. This is not coincidental, since it can be shown that the rate equations of class $B$ laser with externally modulated and re-injected optical signal (see Appendix) are equivalent to ones describing a system with modulated losses.However, our external modulation schemes are much simpler experimentally then the intercavity modulation and thus better suited for a graduate laboratory.

\section{ACKNOWLEDGEMENTS}

This work was supported by the Office of Naval Research (SBIR) under Contract Number N00014-93-C-0051.

\section{APPENDIX}

\subsection{Rate Equations of a Class B Laser with Extemally Modulated and Re-Injected Optical Signal}

$\mathrm{CO}_{2}$ lasers belong to the so-called B-class of lasers where the polarization variable has the shortest relaxation time $\gamma_{\perp}^{-1}$ of the system. The relaxation time $k^{-1}$ of the field variable is typically three orders of magnitude longer than $\gamma_{\perp}{ }^{-1}$ in this class of lasers, whereas the relaxation time $\gamma_{I}^{-1}$ of the population inversion variable sets up the longest intrinsic time scale, that is $\gamma_{I}^{-1} \geq k^{-1} \| \gamma_{\perp}^{-1}$. This hierarchy of relaxation times allows the adiabatic elimination of the polarization variable from the Maxwell-Bloch equations ${ }^{12}$. In the single mode case, these systems are reasonably well described by the rate equations for the field amplitude $E$ and the population inversion $\Delta N$ : 


$$
\frac{d E}{d t}=-k E\left(1+i \theta^{\prime}\right)+\frac{g^{2}}{\gamma_{\perp}} \frac{\Delta N E}{1+i \delta^{\prime}}+k r E \exp [i \varphi(t)]
$$

$$
\frac{d(\Delta N)}{d t}=-\gamma_{\|}\left(\Delta N-\Delta N_{\circ}\right)-2 \frac{2 g^{2}}{\gamma_{\perp}} \frac{1}{1+\delta^{\prime 2}}|E|^{2} \Delta N
$$

where $r$-is the reflection coefficient of an external mirror; $r E \exp [i \varphi(t)]$-represents re-injected field with time depending phase $\varphi(t)$; $\delta^{\prime}=\left(\omega_{o}-\omega_{L}\right) / \gamma_{\perp} ; \theta^{\prime}=\left(\omega_{c}-\omega_{L}\right) / k ; \omega_{c}, \omega_{o}$, and $\omega_{L}$ are the cavity, atomic, and laser operational frequencies, respectively.

Equations for the light intensity $I$ and population inversion $\Delta N$ can be obtained from eq. (1) and (2) assuming that time depending phase $\varphi(t)$ can be described as $\varphi(t)=\varphi_{o}+\varphi_{m} \cos \Omega 2 t$, where: $\varphi_{o}$-constant part of phase; $\varphi_{m}$ - phase modulation amplitude; $\Omega$-modulation frequency.

$$
\frac{d I}{d t}=G I \Delta N-I k_{0}[1+m \cos (\Omega t)]
$$

$$
\frac{d \Delta N}{d t}=-\gamma_{\|}\left(\Delta N-\Delta N_{o}\right)-2 G I \Delta N,
$$

where : $G=2 g^{2} /\left[\gamma_{\perp}\left(1+\delta^{2}\right)\right] ; \quad k_{o}=2 k\left(1-r \cos \varphi_{0}\right)$; $m=\left(r \varphi_{m} \sin \varphi_{d}\right) /\left(1-r \cos \varphi_{0}\right)$.

Equations (3) and (4) are identical to appropriate equations for light intensity and population inversion for system with modulated losses ${ }^{1}$. 


\section{REFERENCES}

1. F.T. Arecchi, R. Meucci, G. Puccioni, and J. Tredicce,"Experimental Evidence of Subharmonic Bifurcations, Multistability, and Turbulence in a Q-Switched Gas Laser," Phys. Rev. Lett. Vol. 49,pp. 1217-1220, 1982.

2. D. Dangoisse, P. Glorieux, and D. Hennequin,"Laser Chaotic Attractors in Crisis," Phys. Rev. Lett.Vol. 57,pp. 2657-2660,1986.

3. J.R. Tredicce, F.T. Arecchi, G.P. Puccioni, A. Foggi, and W. Gadomski, "Dynamic behavior and onset of low-dimensional chaos in modulated homogeneously broadened single-mode laser : Experiments and theory," Physical Review A, Vol.34, 2073-2081,1986.

4. D. Dangoisse, P. Glorieux, and D. Hennequin, Physical Review A,"Chaos in a $\mathrm{CO}_{2}$ laser with modulated parameters: Experiments and numerical simulations," Vol. 36,pp. 4775- 4791, 1987.

5. D.J. Biswas, V.Dev, and U.K. Chatterjee,"Experimental observation of oscillatory instabilities and chaos in a gain-modulated single-mode $\mathrm{cw} \mathrm{CO}_{2}$ laser," Phys. Rev. A, Vol. 35,pp. 456-458,1987.

6. A.V. Bondarenko, A.F. Glova, S.N. Kozlov, F.V. Lebedev, V.V. Likhanskii, A.P. Napartovich, V.D. Pismennii and V.P. Yartsev,"Regular and chaotic pulsation intensity in a $\mathrm{CO}_{2}$-lasers with modulated parameters," Laser Noise, SPIE Proceedings, Vol.1376, pp.117-127,1990.

7. F.T. Arecchi, W. Gadomski and R. Meucci,"Generation of chaotic dynamics by feedback on a laser," Phys. Rev. A ,Vol.34,pp. 1617-1620, 1986.

8. F.T. Arecchi, Chapt. 2 in Instabilities and Chaos in Quantum Optics, F.T.Arecchi and R.G. Harrison, Eds., Springer-Verlag, Berlin, Heidelberg 1987.

9. F.T. Arecchi, G. Giacomelli, A. Lapucci, and R. Meucci, "Dynamics of a $\mathrm{CO}_{2}$ laser with delayed feedback: The short-delay regime," Phys. Rev. A,Vol.43, pp. 4997-5003,1991.

10. A.V. Bondarenko, A.F. Glova, S.N. Kozlov, F.V. Lebedev, V.V. Likhanskii, A.P. Napartovich, V.D. Pismennii and V.P. Yartsev,"Bifurcation and chaos in a system of optically coupled $\mathrm{CO}_{2}$ lasers," Sov. Phys. JETP,Vol. 68,pp. 461466, 1989.

11. V.V. Likhanskii and A.P. Napartovich,"Radiation emitted by optically coupled lasers," Sov. Phys. Usp.Vol. 33,pp. 228-250,1990.

12. J.R.Tredicce,F.T.Arecchi,G.L.Lippi, and G.R.Puccioni," Instabilities in lasers with an injected signal," Journal Optical Society of America B,Vol. 2,pp.173183,1985 . 